01

\title{
Особенности возбуждения линий главной серии атомов подгруппы цинка электронным ударом. І. Кадмий
}

\author{
(C) Г.Г. Богачев, Е.Ю. Ремета \\ Институт электронной физики НАН Украины, \\ 88017 Ужгород, Украина \\ e-mail: bogach.gen@gmail.com, remetoveyu@gmail.com
}

Поступила в редакцию 11.09.2019 г.

В окончательной редакции 29.10.2019 г.

Принята к публикации 01.11.2019 г.

\begin{abstract}
С использованием техники пересекающихся пучков медленных электронов и атомов кадмия измерены функции возбуждения трех спектральных линий его главной серии $(166.9,152.7,146.9 \mathrm{~nm})$, исходящих с уровней $5 \operatorname{sn} p^{1} P_{1}^{o}(n=6,7,8$ соответственно). В диапазоне энергий электронов $12-18 \mathrm{eV}$ на этих функциях обнаружено проявление послестолкновительного взаимодействия медленных рассеянных электронов и быстрых электронов, испущенных при распаде автоионизационных состояний. Этот процесс приводит при энергиях налетающих электронов $\sim 11.8, \sim 12.4$ и $\sim 16.6 \mathrm{eV}$ к дополнительному заселению исходных уровней спектральных переходов и соответственно к максимумам на функциях возбуждения вследствие захвата на эти возбужденные уровни рассеянного электрона. Установлены термы автоионизационных состояний атома, ответственных за наблюдаемые максимумы на функциях возбуждения спектральных линий. В классическом приближении двумя способами - прямым вычислением и аппроксимацией по методу наименьших квадратов - проведена оценка эффективных ширин электронного распада автоионизационных состояний, суммарное действие которых приводит к энергетическому сдвигу максимумов. Использованы приближенные формулы расчета, справедливые для различных соотношений между послестолкновительным сдвигом максимумов на функциях возбуждения и энергией связи атомных уровней.
\end{abstract}

Ключевые слова: столкновения электронов с атомами, возбуждение, вакуумный ультрафиолет, автоионизационное состояние, послестолкновительное взаимодействие.

DOI: $10.21883 /$ OS.2020.02.48957.266-19

\section{Введение}

Спектры поглощения в области вакуумного ультрафиолета (ВУФ) для атомов II группы обнаруживают довольно большие вероятности двухэлектронных переходов из валентной подоболочки $n s^{2}$. Для атомов $\mathrm{Zn}$, $\mathrm{Cd}$ и $\mathrm{Hg}$ (IIB подгруппа) с ними сравнимы также и вероятности одноэлектронных переходов из субвалентной подоболочки $(n-1) d^{10}(n=4,5,6$ соответственно) [1]. В таких переходах возбуждается большое число состояний, лежащих над порогами однократной ионизации атомов и называемых автоионизационными состояниями (АИС). В столкновениях электронов с атомами кадмия АИС возбуждаются весьма эффективно. Из спектров поглощения и спектров испущенных электронов [2] найдено и идентифицировано множество АИС, расположенных до энергий на $\sim 10 \mathrm{eV}$ выше потенциала ионизации (ПИ), I $=8.991 \mathrm{eV}$. Самые низкие принадлежат электронным конфигурациям $5 p^{2}$ и $4 d^{9} 5 s^{2} 5 p$ (см. диаграмму уровней на рис. 1, а также энергетические положения АИС на рис. 2) [2,3]. В работе [4] с использованием метода потерь энергии электронов также было исследовано возбуждение АИС конфигурации $4 d^{9} 5 s^{2} 5 p$ атома кадмия.
Функции возбуждения (ФВ) спектральных линий Cd I в видимой и ультрафиолетовой областях, исходящих с нижних уровней конфигураций $4 d^{10} 5 s n l \quad(n=5-8$; $l=s, p, d)$, были измерены при столкновениях атомов кадмия с электронами с разрешением по энергии $80 \mathrm{meV}$ [5]. Для линий резкой $(n=6-8)$ и диффузной $(n=5,6)$ серий на ФВ были обнаружены четкие максимумы в области расположения АИС, которые были приписаны уровням короткоживущих отрицательных ионов, образованных на АИС конфигурации $4 d^{9} 5 s^{2} 5 p$. Позднее измерения ФВ спектральных линий, испущенных из уровней $4 d^{10} 5 s n s{ }^{1} S_{0}(n=6-11)$, были выполнены с улучшенным энергетическим разрешением (50 meV) [6]. Результаты показали богатую структуру, которая была объяснена двумя различными механизмами дополнительного заселения исходных уровней спектральных линий. С одной стороны, это формирование и распад короткоживущих состояний отрицательных ионов с заселением исходных атомных уровней. Структуры этого рода наблюдаются при одних и тех же энергиях электронов на ФВ спектральных линий, исходящих с разных исходных уровней. С другой стороны, это процесс послестолкновительного взаимодействия (ПСВ) [7]. В результате ПСВ происходит дополнительное заселе- 


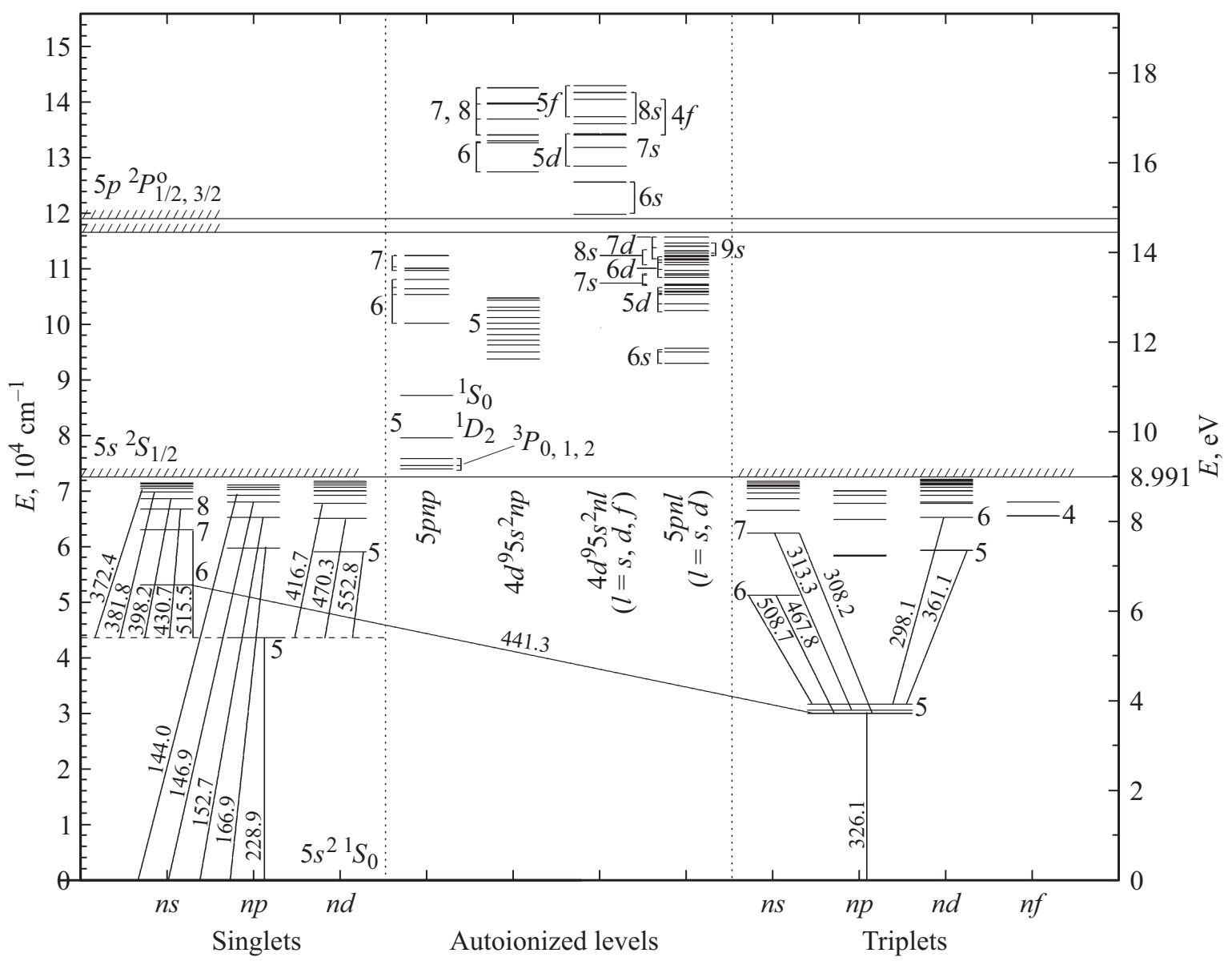

Рис. 1. Диаграмма уровней атома кадмия.

ние атомных уровней в два этапа. Сначала - промежуточный столкновительный процесс возбуждения атома в АИС налетающим электроном. Далее - электронный распад АИС и последующее кулоновское взаимодействие между медленным рассеянным и быстрым испущенным электронами в поле иона приводят к заселению атомных уровней. Возникающие при этом особенности на ФВ спектральных линий, исходящих с этих уровней, оказываются сдвинутыми относительно положений АИС в сторону бо́льших энергий. Этот сдвиг тем больше, чем выше расположен исходный уровень линии (меньше его энергия связи) [7].

Отметим, что подробный теоретический обзор эффекта ПСВ в различных процессах фотон-атомных, атоматомных и электрон-атомных столкновений дан в обзоре [8]. Главным процессом, в котором анализируется проявление этого взаимодействия, является ионизация. Для процесса возбуждения атомных состояний электроном также отмечается роль ПСВ, в частности, при энергиях электрона, превышающих потенциал ионизации.

В работах $[9,10]$ были исследованы ФВ спектральных линий двух серий $\mathrm{Cd}$, исходящих с уровней $5 s n s{ }^{1} S_{0}$ $(n=6-11)$ и $5 s n d^{3} D_{1,2,3} \quad(n=5$ и 6) на синглетные уровни $5 s 5 p^{1} P_{1}^{o}$ и триплетные уровни $5 s 5 p^{3} P_{2}^{o}$ соответ- ственно. Эксперименты были выполнены с разрешением по энергии $\Delta E_{1 / 2}<50 \mathrm{meV}$ для энергий электронов выше порога однократной ионизации (см. также $[6,11])$. Были обнаружены и детально исследованы структуры на ФВ спектральных линий, связанные с ПСВ. В [11] даны результаты идентификации АИС, которые проявляются через ПСВ на ФВ шести спектральных линий Cd I, исходящих с уровней $5 s n s{ }^{1} S_{0}$, а в $[9,10]$ оценены энергии и ширины электронного распада АИС.

Отметим, что в [9-11], как и в [5,6], заметные проявления ПСВ были обнаружены при исследовании ФВ спектральных линий Cd I в видимой и УФ областях. Наиболее интенсивные линии атома кадмия и других атомов подгруппы цинка, а также атома магния (принадлежащие главным сериям) лежат в области ВУФ, но ФВ для них практически не исследованы. В связи с этим нами были начаты работы с целью восполнить указанный пробел. В частности, для магния [12] исследованы ФВ линий главной серии $(n=4-7)$ при столкновениях атомов с электронами энергией до $25 \mathrm{eV}$ и было обнаружено значительное проявление ПСВ вблизи АИС $3 p 4 s{ }^{1} P_{1}^{o}$.

В настоящей работе представлены результаты исследования ФВ для трех линий главной серии атома кадмия, лежащих в ВУФ области. Эти линии исходят с уровней 
атома $5 s n p^{1} P_{1}^{o}$ и оканчиваются на основном уровне $5 s^{2} S_{0}$. Исследованы линии серии для $n=6,7,8$, длины волн $166.9,152.7,146.9 \mathrm{~nm}$ соответственно. Заметим здесь, что первый член серии $(n=5)$, резонансная линия с длиной волны $228.9 \mathrm{~nm}$, нами не исследовался. Предварительные результаты по возбуждению спектральных линий атома кадмия в ВУФ области были представлены на конференциях $[13,14]$. Там сообщалось об исследовании ФВ двух линий главной серии атома $\mathrm{Cd}(n=6,7)$ с длинами волн 166.9 и $152.7 \mathrm{~nm}$ соответственно. Разброс по энергии пучка возбуждающих электронов составлял $\sim 0.7 \mathrm{eV}$. Слабо выраженная особенность при $\sim 10 \mathrm{eV}$ (выше ПИ на $\sim 1 \mathrm{eV}$ ) авторами трактовалась как возможное проявление состояния $5 p^{3}$ отрицательного иона $\mathrm{Cd}$. Лишь два максимума на ФВ этих спектральных линий, обязанные одному или группе близких по энергии АИС, наблюдаются при энергиях $\sim 12.75 \mathrm{eV}(n=6)$ и $13.0 \mathrm{eV}(n=7)$. Таким образом, возможным в этом случае является возбуждение и электронный распад АИС конфигураций $5 p n p, 4 d^{9} 5 s^{2} 5 p$ и 5 pnd.

Отметим, что прямое возбуждение исходных уровней $5 s n p{ }^{1} P_{1}^{o}$ из основного состояния атома, исследуемое в настоящей работе, эффективно происходит в дипольном переходе без изменения спина. Прямое возбуждение уровней $5 s n s{ }^{1} S_{0}$ и $5 s n d^{3} D_{1,2,3}$, которое исследовалось в работах $[9,10]$, происходит по монопольному (без изменения спина) и квадрупольному (с изменением спина) переходам, т.е. такие переходы не являются самыми вероятными. Таким образом, при менее эффективном прямом возбуждении особенности на ФВ спектральных линий, обязанные непрямому каналу заселения уровней, должны проявляться значительно лучше. Это также достаточно хорошо видно на ФВ спектральных линий с уровней $5 \operatorname{snp}{ }^{1} P_{1}^{o}$ для $n>6$, которые возбуждаются менее вероятно, чем резонансная спектральная линия с уровня $5 s 5 p^{1} P_{1}^{o}$.

\section{О технике и методике эксперимента}

Экспериментальная установка, использованная в работе, была в основном подобна описанной в [15] и позже в [16]. Она включала в себя источник излучения и спектрометр с фотоэлектрической регистрацией. В спектрометре был использован монохроматор, собранный нами по оптической схеме Сейа-Намиока. Диспергирующим элементом монохроматора служила вогнутая тороидальная дифракционная решетка для ВУФ области, изготовленная на слое алюминия и защищенная слоем фтористого магния. Радиусы кривизны $R_{m}=500 \mathrm{~mm}$ и $R_{s}=333 \mathrm{~mm}$, плотность нарезки -1200 lines $/ \mathrm{mm}$. Источником излучения служили электронный и атомный пучки, пересекающиеся под прямым углом. В качестве источника пучка электронов использовалась электронная пушка с ленточным оксидным катодом и тремя анодами в виде плоских диафрагм с прямоугольными от- верстиями $(1 \times 8 \mathrm{~mm})$, которая формировала ленточный электронный пучок с плотностью тока $\sim 10^{-3} \mathrm{~A} / \mathrm{cm}^{2}$. В области энергий 6-10 eV ширина энергетического распределения электронов на полувысоте была $\sim 0.6 \mathrm{eV}$. Для получения атомного пучка использовался источник эффузионного типа с резистивным нагревом. Пучок формировался эффузионным каналом круглого сечения диаметром $1 \mathrm{~mm}$ и длиной $8 \mathrm{~mm}$. Концентрация атомов в области столкновений не превышала $\sim 10^{12} \mathrm{~cm}^{-3}$.

В качестве детектора излучения использовался солнечнослепой фотоэлектронный умножитель, который работал в режиме счета фотонов. Процедура измерений и накопления данных была автоматизирована. Для этого был использован IBM-совместимый персональный компьютер с интерфейсными картами ввода-вывода и цифроаналогового преобразователя. Для измерительной системы нами было разработано программное обеспечение на языке Pascal.

Отметим, что для привязки получаемых в эксперименте ФВ спектральных линий к шкале энергий использовалась процедура подгонки их припороговых участков по методу наименьших квадратов с применением алгоритма Левенберга-Марквардта, как описано в [17]. Погрешность определения положения максимумов на ФВ была порядка $0.07 \mathrm{eV}$.

\section{Результаты и обсуждение}

\section{Результаты измерений}

На рис. 1 приведена энергетическая диаграмма уровней нейтрального атома кадмия, где показаны основные спектральные переходы Cd I, а также известные атомные АИС [2], лежащие ниже и выше первого возбужденного состояния $4 d^{10} 5 p^{2} P^{o}$ иона $\mathrm{Cd}^{+}$с энергиями уровней $14.462\left({ }^{2} P_{1 / 2}^{o}\right)$ и $14.770 \mathrm{eV}\left({ }^{2} P_{3 / 2}^{o}\right)$ [3]. Как отмечалось выше, эти АИС принадлежат электронным конфигурациям $5 \mathrm{pnl}(n=5,6,7 ; l=s, p, d)$ и $4 d^{9} 5 s^{2} n l(n=5-9$; $l=s, p, d, f)$ (рис. 2).

На рис. 2 представлены измеренные ФВ спектральных линий $166.9,152.7$ и $146.9 \mathrm{~nm}$, которые отвечают переходам $5 s n p^{1} P_{1}^{o} \rightarrow 5 s^{2}{ }^{1} S_{0} \quad(n=6,7,8$ соответственно), и указаны энергетические положения, до $18 \mathrm{eV}$, АИС, образованных возбуждением двух валентных электронов или одного электрона из субвалентной подоболочки $4 d^{10}$ (рис. 1). Для конфигурации $4 d^{9} 5 s^{2} 5 p$ вертикальными отрезками большей длины отмечены уровни с полным моментом $J=1\left({ }^{3} P,{ }^{1} P,{ }^{3} D\right)$.

Процесс прямого возбуждения исходного уровня спектральных линий при столкновениях атома кадмия с электроном может быть представлен схемой

$$
\mathrm{e}^{-}+\mathrm{Cd}\left(5 s^{2}{ }^{1} S_{0}\right) \rightarrow \mathrm{Cd}\left(5 s n p^{1} P_{1}^{o}\right)+\mathrm{e}_{\mathrm{sc}}^{-}
$$

где $\mathrm{e}_{\mathrm{sc}}^{-}-$рассеянный электрон.

Полученные ФВ (рис. 2) показывают четкие особенности в виде более или менее выраженных максимумов 


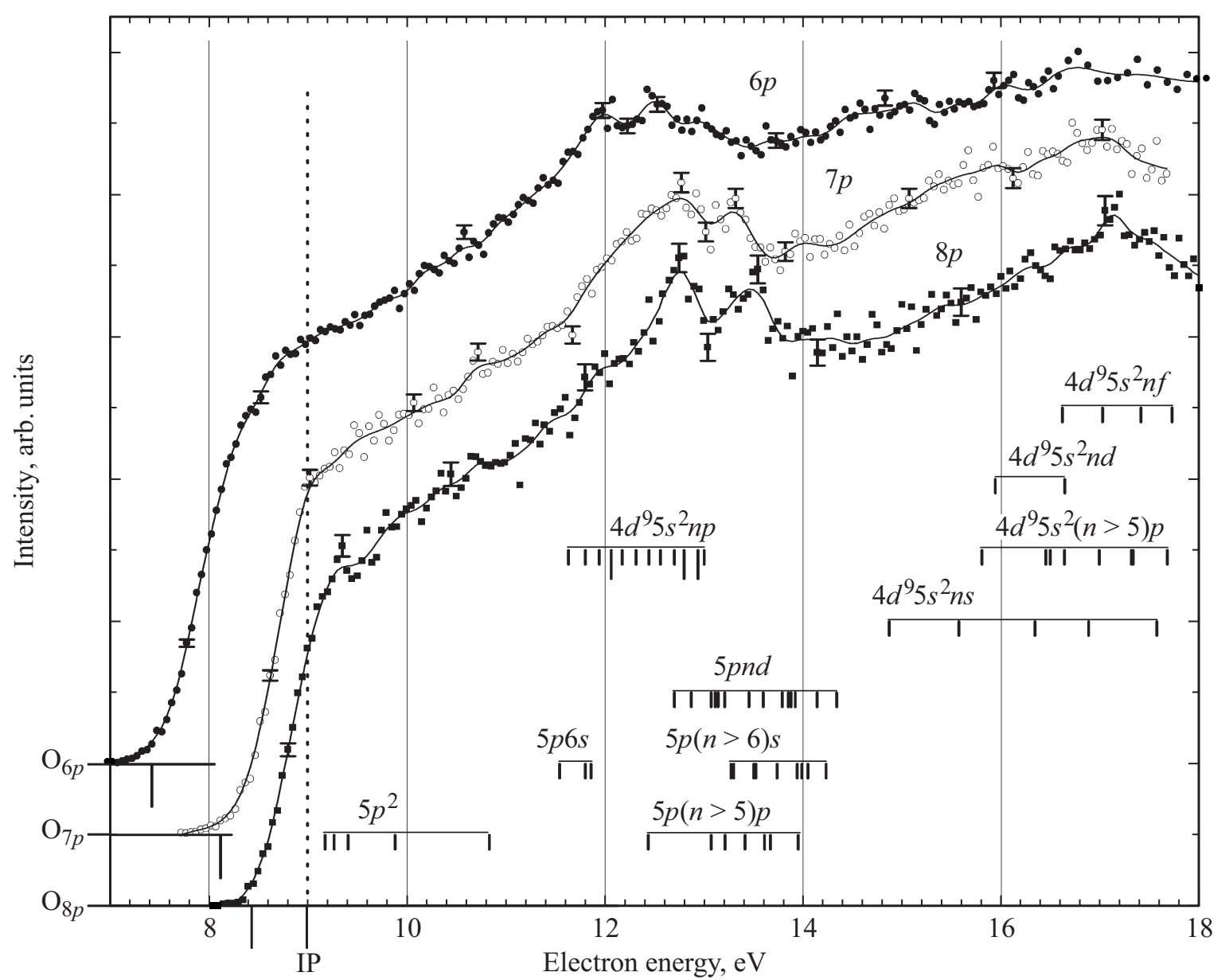

Рис. 2. Функции возбуждения спектральных линий главной серии $\mathrm{Cd}$ I $166.9,152.7,146.9 \mathrm{~nm}\left(\right.$ переходы $5 s n p^{1} P_{1}^{o} \rightarrow 5 s^{2} S_{0}$, $n=6,7,8$ соответственно) и энергетические положения АИС атома [2]. Сплошной линией показан результат сглаживания по пяти точкам данных эксперимента для ФВ.

в области энергий АИС. Интервалы погрешностей измерения $\Phi \mathrm{B}$, показанные на рисунке, соответствуют доверительной вероятности 0.68. Среди них выделяются три особенности на каждой ФВ при следующих значениях энергии электронов: для линии $166.9 \mathrm{~nm}-11.93,12.51$ и $16.78 \mathrm{eV}$; для линии $152.7 \mathrm{~nm}-12.73,13.29$ и $17.02 \mathrm{eV}$; для линии $146.9 \mathrm{~nm}-12.77,13.50$ и $17.13 \mathrm{eV}$. При внимательном рассмотрении ФВ эти особенности были собраны в три группы, представленные в табл. 1. Как видно, в группу 1 включены особенности (максимумы) с наименьшей энергией на каждой ФВ. Максимумы, отнесенные к группам 1 и 2 , выражены заметно сильнее в сравнении с группой 3 (с наибольшей энергией). Они проявляются на более широких структурах $\Phi \mathrm{B}$, которые охватывают энергии АИС конфигураций $5 p 6 s, 4 d^{9} 5 s^{2} 5 p$, 5 pnp $(n>5), 5$ pns $(n>6)$ и 5pnd. Максимумы из группы 3 расположены в энергетической области, где лежат более высокоэнергетические АИС конфигураций $4 d^{9} 5 s^{2} n s, 4 d^{9} 5 s^{2} n p(n>5), 4 d^{9} 5 s^{2} n d$ и $4 d^{9} 5 s^{2} n f$. Все эти конфигурации образованы за счет перехода одного электрона из субвалентной подоболочки $4 d^{10}$ на воз-
Таблица 1. Сведения об исследованных спектральных линиях главной серии $\mathrm{Cd}$ I: энергия возбуждения $E_{\mathrm{ex}}$ и энергия связи $E_{b}$ их исходных уровней, экспериментальные значения энергий $E_{\max }$ максимумов, наблюдаемых на измеренных ФВ

\begin{tabular}{c|c|c|c|c|c|c}
\hline \multirow{2}{*}{$\begin{array}{c}\lambda, \\
\mathrm{nm}\end{array}$} & $\begin{array}{c}\text { Исходный } \\
\text { терм } \\
5 s n p^{1} P_{1}^{o}\end{array}$ & \multirow{2}{*}{$\begin{array}{c}E_{\mathrm{ex}}, \\
\mathrm{eV}\end{array}$} & $\begin{array}{c}E_{b}, \\
\mathrm{eV}\end{array}$ & \multicolumn{3}{|c}{$E_{\max }, \mathrm{eV}$} \\
\cline { 4 - 7 } & & & Группа 1 & Группа 2 & Группа 3 \\
\hline 228.9 & $5 s 5 p$ & 5.416 & 3.575 & 11.77 & 12.26 & 16.64 \\
166.9 & $5 s 6 p$ & 7.425 & 1.566 & 11.93 & 12.51 & 16.78 \\
152.7 & $5 s 7 p$ & 8.118 & 0.873 & 12.73 & 13.29 & 17.02 \\
146.9 & $5 s 8 p$ & 8.435 & 0.556 & 12.77 & 13.50 & 17.13
\end{tabular}

бужденную орбиталь $n l$ в присутствии валентных $5 s^{2}$ электронов.

Положения отмеченных максимумов „сдвигаются“ в сторону бо́льших энергий с ростом главного квантового числа $n$ исходного уровня $5 \operatorname{sn} p^{1} P_{1}^{o}$. Поэтому представляется возможным связать происхождение максимумов на 
ФВ и их „сдвиг“ с эффектом ПСВ [7]. Известно, что свидетельством наличия ПСВ как дополнительного канала заселения атомного уровня является именно то, что при некоторой энергии налетающих электронов $E$, близкой к энергии АИС, наблюдается максимум на ФВ. Этот максимум соответствует наиболее вероятному обмену энергией между рассеянным и испущенным (в результате электронного распада АИС) электронами, с последующим захватом медленного рассеянного электрона в связанное атомное состояние. Энергетическое положение максимума определяется энергией и шириной АИС, а также энергией связи исходного уровня. Меньшей энергии возбуждения уровня (большей энергии связи) соответствует меньший сдвиг. Большей ширине АИС малое время жизни состояния - соответствует больший сдвиг.

Процесс дополнительного заселения исходного уровня спектральных линий с участием АИС, включая его возбуждение, электронный распад и ПСВ между электронами в непрерывном спектре, можно записать в виде следующей схемы:

$$
\begin{aligned}
& \mathrm{e}^{-}+\mathrm{Cd}\left(5 s^{2}{ }^{1} S_{0}\right) \rightarrow \mathrm{Cd}(\mathrm{AИC})+\mathrm{e}_{\mathrm{sc}}^{-\prime} \rightarrow \\
& \rightarrow \mathrm{Cd}^{+}\left(5 s^{2} S_{1 / 2}\right)+\mathrm{e}_{\mathrm{sc}}^{-\prime}+\mathrm{e}_{\mathrm{ej}}^{-} \rightarrow \mathrm{Cd}\left(5 s n p^{1} P_{1}^{o}\right)+\mathrm{e}_{\mathrm{ej}}^{-\prime} .
\end{aligned}
$$

Здесь $\mathrm{e}_{\mathrm{sc}}^{-\prime}-$ электрон, рассеянный после возбуждения АИС, $\mathrm{e}_{\mathrm{ej}}^{-}$- электрон, испущенный при распаде АИС, $\mathrm{e}_{\mathrm{ej}}^{-\prime}$ - испущенный электрон после взаимодействия с рассеянным электроном в поле иона кадмия.

Исходя из отмеченных выше особенностей ПСВ, ответственного за наличие максимумов на $\Phi$, следует ожидать, что энергии электронов, при которых такие максимумы будут иметь место на ФВ линии $166.9 \mathrm{~nm}$ (что соответствует возбуждению исходного уровня $5 s 6 p^{1} P_{1}^{o}$ с наибольшей энергией связи), должны быть близки к энергиям соответствующих АИС с учетом разброса по энергиям электронов в пучке. Так, уровни упомянутых выше автоионизационных конфигураций $5 p 6 s$, 5pnp $(n>5)$, 5pns $(n>6)$ и 5pnd совместно участвуют в образовании широких неразрешенных максимумов из групп 1 и 2. В работе [4] экспериментально установлено, что уровни ${ }^{3} P_{1}^{o}$ (энергия $12.062 \mathrm{eV}$, линия 3 [4]) и ${ }^{1} P_{1}^{o}(12.810 \mathrm{eV}$, линия 4 [4]) автоионизационной конфигурации $4 d^{9} 5 s^{2} 5 p$, а также уровень ${ }^{1} P_{1}^{o}$ автоионизационной конфигурации $4 d^{9} 5 s^{2} 6 p(15.807 \mathrm{eV}$, линия 13 [4]) возбуждаются существенно эффективнее, чем другие уровни этих конфигураций. Вероятно поэтому и максимумы на $Ф \mathrm{~B}$ спектральной линии с уровня $5 s 6 p^{1} P_{1}^{o}$ при $\sim 12$ и $\sim 12.5 \mathrm{eV}$ также обязаны возбуждению уровней автоионизационной конфигурации $4 d^{9} 5 s^{2} 5 p$, а при $\sim 16.8 \mathrm{eV}-$ автоионизационных конфигураций $4 d^{9} 5 s^{2} n p(n>5)$ и $4 d^{9} 5 s^{2} n f$.

Нельзя также исключать возможного образования промежуточных короткоживущих состояний отрицательного иона $\mathrm{Cd}^{-}$(с последующим электронным распадом) при возбуждении автоионизационных уровней конфигурации $4 d^{9} 5 s^{2} 5 p$. Такого рода состояния наблюдали в [5] как весьма выразительные острые пики (резонансы Фешбаха) на ФВ ряда спектральных линий атома при энергиях электронов выше потенциала ионизации. В частности, для линий $515.5,430.7 \mathrm{~nm}$ (переходы $5 s n s^{1} S_{0} \rightarrow 5 s 5 p^{1} P_{1}^{o}$ для $n=7,8$ соответственно) и $361.0,298.0 \mathrm{~nm}$ (переходы $5 s n d^{3} D_{1,2,3} \rightarrow 5 s 5 p^{3} P_{2}^{o}$ для $n=5,6$ соответственно) средняя энергия резонансных пиков составляла $11.83 \mathrm{eV}$, что энергетически близко к некоторым уровням конфигураций $5 p 6 s$ и $4 d^{9} 5 s^{2} 5 p$ (рис. 2).

Укажем и другие особенности, имеющие место на ФВ (рис. 1 и 2). При энергиях электронов, близких к ПИ $(8.991 \mathrm{eV})$, уступовидная особенность при энергиях от 9.20 до $9.46 \mathrm{eV}$ наблюдается на всех ФВ. Ее происхождение, вероятно, связано с короткоживущими состояниями отрицательного иона трехэлектронной конфигурации $5 p^{3}$. Они формируются низколежащими уровнями ${ }^{3} P_{0,1,2}$ (средняя энергия $\sim 9.35 \mathrm{eV}$ ) двухэлектронной автоионизационной конфигурации $5 p^{2}$. Аналогично при энергиях электронов от $\sim 10.57$ до $10.68 \mathrm{eV}$ на всех $Ф \mathrm{~B}$ имеется особенность, которая, вероятно, также может быть связана с состоянием отрицательного иона $\mathrm{Cd}^{-}$ в конфигурации $5 p^{3}$. Однако это состояние формируется уже на автоионизационном уровне ${ }^{1} S_{0}$ (энергия $\sim 10.83 \mathrm{eV}$ ) той же конфигурации $5 p^{2}$. Отметим также, что при энергиях $\sim 9.93-10.04 \mathrm{eV}$ видна очень слабо выраженная особенность, хорошо совпадающая с автоионизационным уровнем $5 p^{2}{ }^{1} D_{2}$ (энергия $\sim 9.9 \mathrm{eV}$ ). По-видимому, ее также можно приписать проявлению состояния $5 p^{3}$ иона $\mathrm{Cd}^{-}$. Все указанные здесь особенности, приписываемые состояниям отрицательного иона кадмия, расположенным в автоионизационной области, не имеют выраженного энергетического сдвига. Это исключает их связь с ПСВ и указывает на то, что они представляют собой резонансы Фешбаха процесса возбуждения уровней $5 \operatorname{sn} p^{1} P_{1}^{o}$.

\section{Результаты теоретических оценок}

Энергетическое положение максимума $E_{\max }=E_{\text {ais }}+E_{1}$ определяется энергией $E_{\text {ais }}$ и шириной $\Gamma_{\text {ais }}$ отдельного АИС и энергией связи $E_{b}$ исходного уровня, на который при ПСВ захватывается электрон. Последняя равна разности энергий ионизации и возбуждения исходного уровня. Сдвиг максимума $E_{1}$, обязанный ПСВ, удовлетворяет следующему уравнению в классическом приближении (все энергетические величины в еV) [7]:

$$
\left(3 E_{1}+E_{b}\right) \Gamma_{\text {ais }}\left(\frac{R}{E_{1}}\right)^{1 / 2}=\left(E_{1}+E_{b}\right)\left(5 E_{1}+E_{b}\right),
$$

$R$ - постоянная Ридберга $(13.6058 \mathrm{eV})$.

При $E_{1} \ll E_{b} / 2$ получаем простое выражение [10]:

$$
E_{1}=\frac{\Gamma_{\text {ais }}^{2} R}{E_{b}^{2}},
$$


или несколько более сложное, но более точное (при $\left.E_{1} \ll E_{b}\right):$

$$
E_{1}=\frac{E_{b}}{A}\left[\left(1+2 A \Gamma_{\text {ais }}^{2} R / E_{b}^{3}\right)^{1 / 2}-1\right],
$$

где $A-$ постоянная, принимающая значения $A=4,8$, 12. При этом большему значению $A$ соответствует более точное приближение.

Формулы (2) и (3) удобно использовать как для прямого вычисления ширин отдельных АИС, так и для проведения аппроксимации по экспериментальным точкам. Также автоионизационные ширины будем находить из уравнения (1). В случае, когда исходый уровень имеет достаточно большую энергию возбуждения (малая энергия связи $\left.E_{b}\right)$ и неравенство $E_{1} \ll E_{b}$ не выполняется, использование для оценок выражений (2) и (3) является достаточно формальным. Аналогично поступают при использовании формулы Баркера-Берри [18] для оценок увеличения энергии испущенного при автоионизационном распаде электрона (равного уменьшению энергии рассеянного электрона) при ПСВ в процессе ионизации атома.

Необходимо иметь в виду, что при плохой монокинетичности пучка налетающих электронов сдвиг положения неразрешенного максимума на $Ф \mathrm{~B}$ с ростом главного квантового числа $n$ исходного уровня спектральной линии является вкладом ПСВ от всех тех АИС, которые попадают в энергетическую ширину пучка. Поэтому автоионизационная ширина, определяемая по сдвигу всего широкого максимума, является некой эффективной шириной, зависящей от суммарного действия указанных АИС. Улучшение монокинетичности электронного пучка приводит к разрешению тонкой структуры широкого максимума, что позволяет установить более однозначное соответствие между ее особенностями и АИС. Поэтому можно считать, что значительный разброс налетающих электронов по энергии $(\sim 0.6 \mathrm{eV})$ в нашем эксперименте приводит к тому, что вклады от ПСВ за счет конкретных АИС не могут быть разрешены. В результате имеем широкие неразрешенные максимумы, их „сдвиг“ по энергии, наблюдаемый на $Ф \mathrm{~B}$, происходит за счет участия именно многих АИС. Энергетическое положение максимума определяется вероятностью возбуждения образующих его АИС.

Выше была отмечена возможная роль автоионизационных конфигураций $4 d^{9} 5 s^{2} 5 p, 4 d^{9} 5 s^{2} n p(n>5)$ и $4 d^{9} 5 s^{2} n f$ в образовании максимумов на ФВ линии $166.9 \mathrm{~nm}$ с уровня $5 s 6 p^{1} P_{1}^{o}$. Наметим возможные АИС, возбуждаемые из основного состояния $5 s^{2} S_{0}$ атома кадмия, с энергиями, не превышающими энергий первых максимумов в группах на $Ф \mathrm{~B}$ линии с уровня $5 s 6 p^{1} P_{1}^{o}[2-4,19]$.

$\mathrm{B}$ частности, максимуму при $11.93 \mathrm{eV}$ могут соответствовать следующие АИС, обязанные переходам одного электрона из субвалентной подоболочки $4 d^{10}$ или двух электронов из валентной оболочки $5 s^{2}$, с энергиями, не превышающими энергии максимума (в скобках доля данного состояния в \%):

$$
\begin{array}{rr}
4 d^{9} 5 s^{2} 5 p-{ }^{3} P_{2}^{o}(90)+{ }^{3} D_{2}^{o}(7)+\ldots, & 11.634 \mathrm{eV}, \\
{ }^{3} F_{3}^{o}(57)+{ }^{1} F_{3}^{o}(35)+\ldots, & 11.804 \mathrm{eV}, \\
4 d^{10} 5 p 6 s-{ }^{3} P_{2}^{o}(99), & 11.804 \mathrm{eV}, \\
{ }^{1} P_{1}^{o}(77)+{ }^{3} P_{1}^{o}(19)+\ldots, & 11.861 \mathrm{eV} .
\end{array}
$$

Средняя энергия этих АИС с учетом их статвесов $2 J+1$ равна $11.770 \mathrm{eV}$. Считаем, что АИС ${ }^{3} P_{0}^{o}(99.6)$ и ${ }^{3} P_{1}^{o}(81)+{ }^{1} P_{1}^{o}(19), 11.544 \mathrm{eV}$, конфигурации $4 d^{10} 5 p 6 s$ не дают прямого вклада в образование максимума, ибо расположены слишком низко.

Максимуму при $12.51 \mathrm{eV}$ могут соответствовать следующие АИС, обязанные переходам одного электрона из субвалентной подоболочки $4 d^{10}$ или двух электронов из валентной оболочки:

$$
\begin{array}{cc}
4 d^{9} 5 s^{2} 5 p-{ }^{3} P_{1}^{o}(81)+{ }^{1} P_{1}^{o}(11)+\ldots, & 12.062 \mathrm{eV}, \\
{ }^{3} F_{2}^{o}(36)+{ }^{1} D_{2}^{o}(31)+\ldots, & 12.175 \mathrm{eV}, \\
{ }^{3} D_{3}^{o}(75)+{ }^{1} F_{3}^{o}(21)+\ldots, & 12.314 \mathrm{eV}, \\
{ }^{3} P_{0}^{o}(97)+4 d^{9}\left(5 p^{3}{ }^{o} P^{o}{ }^{3} P_{0}^{o}(3),\right. & 12.444 \mathrm{eV}, \\
4 d^{10} 5 p 6 p-{ }^{3} D_{1}(65)+{ }^{3} S_{1}(31), & 12.440 \mathrm{eV}
\end{array}
$$

Средняя энергия этих АИС с учетом статвесов $2 J+1$ равна $12.264 \mathrm{eV}$.

И наконец, максимуму при $16.78 \mathrm{eV}$ могут соответствовать следующие АИС, обязанные переходу одного электрона из субвалентной подоболочки $4 d^{10}$ :

$$
\begin{array}{lll}
4 d^{9} 5 s^{2} 4 f- & { }^{3} D_{1}^{o}(59)+{ }^{1} P_{1}^{o}(31), & 16.621 \mathrm{eV}, \\
4 d^{9} 5 s^{2} 7 p- & { }^{1} P_{1}^{o}(57)+{ }^{3} P_{1}^{o}(31), & 16.641 \mathrm{eV}, \\
4 d^{9} 5 s^{2} 5 d- & { }^{3} D_{2}(46)+{ }^{3} P_{2}(27)+\ldots, & 16.644 \mathrm{eV}
\end{array}
$$

Средняя энергия этих АИС с учетом статвесов $2 J+1$ равна $16.637 \mathrm{eV}$.

Как видим, при электронном возбуждении АИС из основного состояния атома происходит следующее: изменение четности, увеличение на 1-3 полного момента, изменение мультиплетности на 1 , увеличение на 1-3 суммарного орбитального момента. Возможно, что наиболее эффективно из намеченных АИС возбуждаются те, энергия которых наиболее близка к указанным выше средним энергиям групп АИС $\left(E_{\mathrm{ej}}=E_{\text {ais }}-I\right.$, энергия электрона, испущенного при распаде АИС):

для максимума $11.93 \mathrm{eV}-4 d^{9} 5 s^{2} 5 p\left({ }^{3} P_{2}^{o}(90)+{ }^{3} D_{2}^{o}(7)\right)$, $11.634 \mathrm{eV}, E_{\mathrm{ej}}=2.643 \mathrm{eV}$,

для максимума $12.51 \mathrm{eV}-4 d^{9} 5 s^{2} 5 p\left({ }^{3} P_{1}^{o}(81)+{ }^{1} P_{1}^{o}(11)\right)$, $12.062 \mathrm{eV}, E_{\mathrm{ej}}=3.071 \mathrm{eV}$,

для максимума $16.78 \mathrm{eV}-4 d^{9} 5 s^{2} 7 p\left({ }^{1} P_{1}^{o}(57)+{ }^{3} P_{1}^{o}(31)\right)$, $16.641 \mathrm{eV}, E_{\mathrm{ej}}=7.650 \mathrm{eV}$.

Отсчитывая сдвиги положений наблюдаемых широких неразрешенных максимумов в каждой группе относительно указанных выше средних энергий АИС, мы тем самым предполагаем, что на ФВ резонансной линии 
(переход с уровня $5 s 5 p^{1} P_{1}^{o}$ ) возможные положения максимумов ПСВ совпали бы с этими средними энергиями (они и приведены в табл. 1 для линии $228.8 \mathrm{~nm}$ ).

В сообщениях $[13,14]$ мы, исходя из энергий максимумов на ФВ, полагали, что они обусловлены [4] АИС $4 d^{9} 5 s^{2} 5 p^{3} P_{1}^{o}(12.062 \mathrm{eV})$ и ${ }^{1} P_{1}^{o}(12.810 \mathrm{eV})$. Как видим, теперь, по нашему мнению, только АИС $4 d^{9} 5 s^{2} 5 p^{3} P_{1}^{o}$ может быть причиной появления максимума при $12.51 \mathrm{eV}$ на ФВ линии с $5 s 6 p^{1} P_{1}^{o}$. В работах $[10,11]$ максимумы на ФВ линии с уровня $5 s 6 s^{1} S_{0}$, нижнего из измеренных уровней $5 s n s^{1} S_{0}(n=6-11)$, наблюдались при энергиях 10.94, 11.64, 11.81, 12.04 и $12.39 \mathrm{eV}$. Предполагалось, что они обусловлены следующими АИС [2]: $5 p^{2}{ }^{1} S_{0}(10.834 \mathrm{eV}), 4 d^{9} 5 s^{2} 5 p^{3} P_{2}^{o}$ $(11.634 \mathrm{eV}), 4 d^{9} 5 s^{2} 5 p^{3} F_{3}^{o}$ или $5 p 6 s^{3} P_{2}^{o}(11.804 \mathrm{eV})$, $4 d^{9} 5 s^{2} 5 p^{3} P_{1}^{o}(12.063 \mathrm{eV}), 4 d^{9} 5 s^{2} 5 p^{3} D_{3}^{o}(12.314 \mathrm{eV})$. Эти же максимумы в работе [10] наблюдались на ФВ линии с уровня $5 s 5 d^{3} D_{1,2,3}$, нижнего из измеренных уровней $5 s n d^{3} D_{1,2,3}(n=5,6)$. В дополнение к ним на ФВ линии с уровня $5 s 5 d^{3} D_{1,2,3}$ был найден еще и максимум при энергии $12.85 \mathrm{eV}$, обусловленный АИС $4 d^{9} 5 s^{2} 5 p^{1} P_{1}^{o}$ $(12.80 \mathrm{eV})$. Как видим, только два АИС: $4 d^{9} 5 s^{2} 5 p^{3} P_{2}^{o}$ с энергией $11.634 \mathrm{eV}$ и $4 d^{9} 5 s^{2} 5 p^{3} P_{1}^{o}$ с энергией $12.063 \mathrm{eV}$, обусловливают максимумы как на ФВ линии с уровня $5 s 6 s^{1} S_{0}[10,11]$, так и на ФВ линии с уровня $5 s 6 p^{1} P_{1}^{o}$ в нашем случае. По-видимому, это следствие того, что в $[10,11]$ разброс пучка возбуждающих электронов по энергии был на порядок меньше, чем в нашей работе.

В работе [4] представлены энергии некоторых АИС, определенные по спектру потерь энергии $(40$ и $60 \mathrm{eV}$ при рассеянии на малые, $5^{\circ}$ и $4^{\circ}$, углы) налетающих электронов. Трем выделенным нами максимумам соответствуют АИС с энергиями 11.605, 12.062, $16.649 \mathrm{eV}$ (линии 1, 3 и 17 в [4]). Отметим, что, согласно приведенному в этой работе спектру, линия 3 имеет весьма большую интенсивность по сравнению с двумя остальными, из которых линия 1 вообще едва заметна.

В табл. 2 приведены энергетические сдвиги положений максимумов трех групп и проведенные по ним оценки эффективных ширин АИС, ответственных за суммарное проявление ПСВ [7-10,12-14]. Эти ширины определены по формулам (2), (3) и из уравнения (1). Эффективные ширины приведены в порядке увеличения точности их определения. Видим, что автоионизационная ширина, полученная из уравнения (1) по максимумам группы 3, лежит в пределах от 0.197 до $0.294 \mathrm{eV}$. При этом в случае 1-го и 2-го максимумов этой группы точные значения (из решения уравнения (1)) удовлетворительно совпадают с приближенными, полученными по формуле (3) с $A=12$. Аналогичное достаточно удовлетворительное совпадение автоионизационных ширин имеется для каждого из максимумов на ФВ линий с уровня $5 s 6 p^{1} P_{1}^{o}$.

На рис. 3 приведены зависимости $E_{1}\left(E_{b}\right)$ сдвигов широких максимумов от энергии связи исходных уровней $5 \operatorname{snp}{ }^{1} P_{1}^{o}(n=6-8)$. Как видим, третий максимум

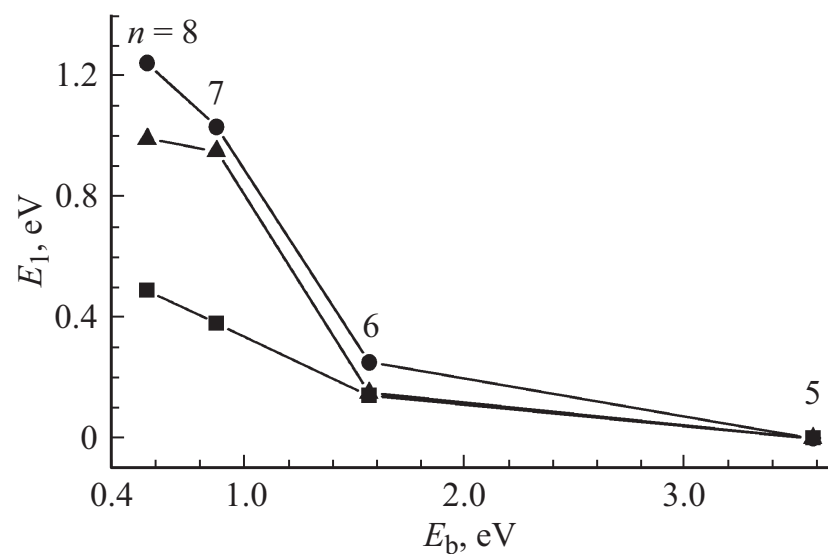

Рис. 3. Зависимости энергетических сдвигов $E_{1}\left(E_{b}\right)$ положений максимумов трех групп на ФВ: $\boldsymbol{\Delta}-1, \bullet-2, \boldsymbol{\square}-3$.

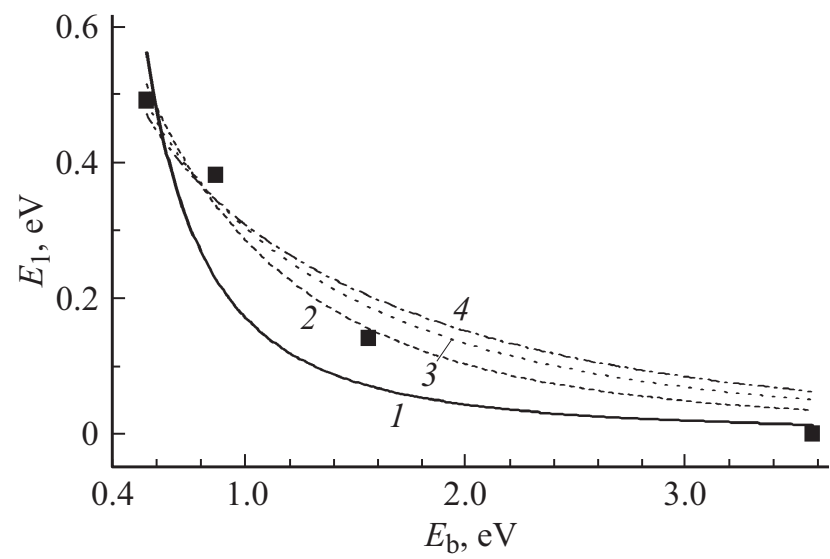

Рис. 4. Аппроксимация энергетических сдвигов $E_{1}\left(E_{b}\right)$ максимумов третьей группы. Кривые, вычисленные по формулам (2) и (3): $1-(2) ; 2-(3), A=4 ; 3-(3), A=8 ; 4-(3), A=12$.

группы 1 на ФВ линии с уровня $5 s 8 p^{1} P_{1}^{o}$ имеет небольшое (на $0.04 \mathrm{eV}$ ) смещение по сравнению со вторым максимумом. Это приводит к некоторому уменьшению эффективной автоионизационной ширины, определяемой по характеристикам $E_{b}$ и $E_{1}$.

Аппроксимация методом наименьших квадратов, проведенная для значений $E_{1}\left(E_{b}\right)$ максимумов трех групп дала следующие оценочные значения эффективных автоионизационных ширин (в $\mathrm{eV}$ ) от действия АИС, ответственных за максимумы каждой группы. Для группы 1 по формуле (2) $\Gamma_{\text {ais }}=0.165 \pm 0.019$, по (3) $A=4-0.332 \pm 0.042, A=8-0.420 \pm 0.067$, $A=12-0.491 \pm 0.088$; для группы 2 по формуле (2) $\Gamma_{\text {ais }}=0.180 \pm 0.017$, по (3) $A=4-0.382 \pm 0.039$, $A=8-0.489 \pm 0.068, \quad A=12-0.573 \pm 0.091$; для группы 3 по формуле (2) $\Gamma_{\text {ais }}=0.113 \pm 0.010$, по (3) $A=4-0.182 \pm 0.008, A=8-0.223 \pm 0.013$, $A=12-0.255 \pm 0.020$.

На рис. 4 на примере сдвигов энергетических положений максимумов группы 3 показаны аппроксимаци- 
Таблица 2. Энергии связи $E_{b}=I-E_{\mathrm{ex}}(\mathrm{eV})$, экспериментальные значения сдвигов по энергии $E_{1}(\mathrm{eV})$ максимумов ФВ и вычисленные эффективные ширины $\Gamma_{\text {ais }}(\mathrm{eV}) \mathrm{AИC}$

\begin{tabular}{|c|c|c|c|c|c|}
\hline Исходный уровень $5 s n p^{1} P_{1}^{o}$ & $E_{b}$ & Расчетная формула & Группа 1 & Группа 2 & Группа 3 \\
\hline $5 s 5 p$ & 3.575 & - & $E_{1}=0.0$ & $E_{1}=0.0$ & $E_{1}=0.0$ \\
\hline $5 s 6 p$ & 1.566 & 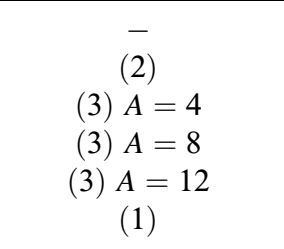 & $\begin{aligned} E_{1} & =0.16 \\
\Gamma_{\text {ais }} & =0.170 \\
\Gamma_{\text {ais }} & =0.186 \\
\Gamma_{\text {ais }} & =0.202 \\
\Gamma_{\text {ais }} & =0.216 \\
\Gamma_{\text {ais }} & =0.216\end{aligned}$ & $\begin{array}{c}E_{1}=0.25 \\
0.212 \\
0.244 \\
0.272 \\
0.297 \\
0.299\end{array}$ & $\begin{array}{c}E_{1}=0.14 \\
0.159 \\
0.172 \\
0.185 \\
0.197 \\
0.197\end{array}$ \\
\hline $5 s 7 p$ & 0.873 & $\begin{array}{c}\text { (2) } \\
\text { (3) } A=4 \\
\text { (3) } A=8 \\
\text { (3) } A=12 \\
\text { (1) }\end{array}$ & $\begin{array}{c}E_{1}=0.96 \\
\Gamma_{\text {ais }}=0.232 * \\
\Gamma_{\text {ais }}=0.415 * \\
\Gamma_{\text {ais }}=0.539 * \\
\Gamma_{\text {ais }}=0.639 * \\
\Gamma_{\text {ais }}=0.736\end{array}$ & $\begin{array}{c}E_{1}=1.03 \\
0.240^{*} \\
0.440^{*} \\
0.575^{*} \\
0.683^{*} \\
0.796\end{array}$ & $\begin{array}{c}E_{1}=0.38 \\
0.146 \\
0.200 \\
0.242 \\
0.277 \\
0.288\end{array}$ \\
\hline $5 s 8 p$ & 0.556 & $\begin{array}{c}\text { (2) } \\
\text { (3) } A=4 \\
\text { (3) } A=8 \\
\text { (3) } A=12 \\
\text { (1) }\end{array}$ & $\begin{array}{c}E_{1}=1.00 \\
\Gamma_{\text {ais }}=0.151 * \\
\Gamma_{\text {ais }}=0.323 * \\
\Gamma_{\text {ais }}=0.431 * \\
\Gamma_{\text {ais }}=0.517 * \\
\Gamma_{\text {ais }}=0.659\end{array}$ & $\begin{array}{c}E_{1}=1.24 \\
0.168^{*} \\
0.392^{*} \\
0.529^{*} \\
0.636^{*} \\
0.857\end{array}$ & $\begin{array}{c}E_{1}=0.49 \\
0.105^{*} \\
0.175^{*} \\
0.224^{*} \\
0.265^{*} \\
0.294\end{array}$ \\
\hline
\end{tabular}

Примечание. * Эффективные ширины вычислены с использованием выражений (2) и (3), когда неравенство $E_{1} \ll E_{b}$ не выполняется.

онные кривые $E_{1}\left(E_{b}\right)$, проведенные с использованием формул (2) и (3) (см. также табл. 2, рис. 3). Сопоставляя значения $\Gamma_{\text {ais }}$ этой группы с данными табл. 2, видим, что по формуле (2) аппроксимационная эффективная ширина оказывается меньшей по величине. Она же, полученная по формуле (3), примерно равна среднему значению от всех ширин, вычисленных при данном значении А. Этот же вывод справедлив для максимумов групп 1 и 2.

Таким образом все намеченные нами выше АИС для максимумов групп 1, 2 и 3 суммарно распадаются путем двухэлектронного перехода в основное состояние $4 d^{10} 5 s^{2} S_{1 / 2}$ иона $\mathrm{Cd}^{+}$с достаточно большими $\sim 0.66-0.74$ и $\sim 0.80-0.86 \mathrm{eV}$ и умеренной, до $\sim 0.3 \mathrm{eV}$, эффективными ширинами соответственно. Дальнейшее ПСВ в непрерывном спектре приводит к передаче энергии от медленного рассеянного электрона, с учетом немонокинетичности, к медленным (с энергиями порядка 2.64 и $3.07 \mathrm{eV})$ или быстрым $(7.65 \mathrm{eV})$ испущенным электронам и последующему захвату рассеянного электрона в связанные состояния $5 \operatorname{sn}{ }^{1} P_{1}^{o}$.

В работах $[13,14]$ на основании предварительных данных по энергиям максимумов, приписываемых эффекту ПСВ, с использованием описанной выше процедуры аппроксимации были получены оценки автоионизационных ширин для АИС $4 d^{9} 5 s^{2} 5 p^{3} P_{1}^{o}$ и ${ }^{1} P_{1}^{o}-0.34$ и $0.38 \mathrm{eV}$ соответственно. Настоящие измерения дали несколько отличающиеся результаты по энергиям наблюдаемых максимумов на ФВ линий главной серии атома кадмия, что привело к иному определению связанных с ними АИС, что было отмечено выше. Соответственно изменились и оценки эффективных автоионизационных ширин от действия АИС.

В работе $[10]$ для заселения $5 s n s{ }^{1} S_{0} \quad(n=6-11)$ уровней через АИС $4 d^{9} 5 s^{2} 5 p^{3} P_{2}^{o}$ и ${ }^{3} P_{1}^{o}$ по формуле (3) с $A=12$ и из уравнения (1) получены следующие автоионизационные ширины: от 0.058 до $0.095 \mathrm{eV}$ (по аппроксимации $0.052 \mathrm{eV}$ ); от 0.142 до $0.212 \mathrm{eV}$ (по аппроксимации $0.138 \mathrm{eV})$. Автоионизационные ширины этих же АИС, заселяющих уровни $5 s n d^{3} D_{1,2,3}(n=5,6)$ посредством процесса ПСВ, полученные по таким же оценкам - по формуле (3) с $A=12$ и из уравнения (1) - меньше и составляют соответственно от 0.064 до $0.078 \mathrm{eV}$ и от 0.042 до $0.045 \mathrm{eV}$. Как видим, полученные нами в настоящей работе эффективные (как результат действия многих АИС максимумов групп 1 и 2) автоионизационные ширины $-\sim 0.7$ и $\sim 0.8 \mathrm{eV}-$ только качественно совпадают с приведенными в [10] (для первого АИС они меньше, чем для второго), а количественно они значительно их превосходят.

Отметим, что оба АИС $\left(4 d^{9} 5 s^{2} 5 p^{3} P_{2}^{o}\right.$ и $\left.{ }^{3} P_{1}^{o}\right)$ участвуют в непрямом заселении уровней $5 s n s^{1} S_{0}$ $(n=6-11)[9,10]$ и $5 s n d^{3} D_{1,2,3}(n=5,6)$ [10]. В этом плане наши наблюдения особенностей на ФВ линий с уровней $5 s n p^{1} P_{1}^{o}(n=6-8)$ дополняют указанные измерения. Таким образом, непрямым путем через АИС происходит заселение уровней с различными четностями, одноэлектронным и полным орбитальными моментами. 
Распад первого АИС:

$$
4 d^{9} 5 s^{2} 5 p^{3} P_{2}^{o}+\mathrm{e}_{\mathrm{sc}}^{-} \rightarrow 4 d^{10} 5 s^{2} S_{1 / 2}+\mathrm{e}_{\mathrm{ej}}^{-}+\mathrm{e}_{\mathrm{sc}}^{-}
$$

дает нечетные значения орбитального момента испущенного электрона: $l_{\text {ej }}=1,3$. При этом медленный рассеянный электрон имеет малые моменты: $l_{\mathrm{sc}}=0,1,2,3$. Распад второго АИС:

$$
4 d^{9} 5 s^{2} 5 p^{3} P_{1}^{o}+\mathrm{e}_{\mathrm{sc}}^{-} \rightarrow 4 d^{10} 5 s^{2} S_{1 / 2}+\mathrm{e}_{\mathrm{ej}}^{-}+\mathrm{e}_{\mathrm{sc}}^{-}
$$

дает для орбитального момента испущенного электрона только одно значение $l_{\text {ej }}=1$. Обмен энергией между электронами $\mathrm{e}_{\mathrm{sc}}^{-}$и $\mathrm{e}_{\mathrm{ej}}^{-}$при ПСВ и захват рассеянного электрона на четные $5 s n s{ }^{1} S_{0}, 5 s n d{ }^{3} D_{1,2,3}$ и нечетные $5 s n p^{1} P_{1}^{o}$ исходные уровни может приводить к изменению четности испущенного электрона при нечетных и четных значениях $l_{\mathrm{sc}}$ соответственно. Тем самым при данном ПСВ для приведенных АИС и заселяемых состояний изменяется орбитальный момент $l_{\mathrm{ej}}$.

\section{Заключение}

Для трех наиболее интенсивных ВУФ линий главной серии CdI 166.9, 152.7 и $146.9 \mathrm{~nm}$ с использованием пересекающихся электронного и атомного пучков были исследованы ФВ при энергиях электронов от порога возбуждения до $\sim 18 \mathrm{eV}$.

На измеренных ФВ были впервые обнаружены три группы широких максимумов при энергиях электронов больше ПИ, появление и смещение которых с ростом главного квантового числа объясняется процессом ПСВ. При этом максимумы при малых энергиях обусловлены уровнями конфигураций $4 d^{10} 5 p n l(n>5, l=0,1)$, сходящихся к порогам возбуждения состояний $4 d^{10} 5 p^{2} P^{o}$ иона $\mathrm{Cd}^{+}$и $4 d^{9} 5 s^{2} 5 p$, а при бо́льших - уровнями более высоковозбужденных конфигураций $4 d^{9} 5 s^{2} n l$ $(n>5, l=0-3)$, сходящихся к возбужденным состояниям $4 d^{9} 5 s^{2}{ }^{2} D$ иона $\mathrm{Cd}^{+}$. По приближенным формулам, количественно описывающим это взаимодействие в классическом приближении, оценены эффективные ширины электронного распада АИС атома кадмия для каждого максимума в группе. Суммарное действие этих АИС ответственно за широкие максимумы и приводит к их энергетическому сдвигу. Получены значения автоионизационных ширин порядка сотен миллиэлектронвольт, что связано с большими значениями измеренных сдвигов положений максимумов ФВ. Эффективные автоионизационные ширины также оценены с использованием указанных формул в процедуре аппроксимации измеренных сдвигов максимумов по энергии в каждой из групп. Полученные таким образом ширины несколько меньше, чем при прямом вычислении, и примерно равны среднему значению ширин, найденных по приближенным формулам. Указано на возможность передачи орбитального момента при ПСВ между рассеянным и испущенным электронами, которая может быть достаточно эффективна при малых энергиях частиц.
Несмотря на невысокое энергетическое разрешение $(\sim 0.6 \mathrm{eV})$ в области локализации первой автоионизационной конфигурации атома кадмия $5 p^{2}$, ближайшей к ПИ, на исследованных ФВ впервые наблюдался ряд особенностей при $\sim 9.20-9.46, \sim 9.93-10.04$ и $\sim 10.57-10.68 \mathrm{eV}$. Их появление объясняется процессами формирования на автоионизационных уровнях ${ }^{3} P_{0,1,2},{ }^{1} D_{2},{ }^{1} S_{0}$ этой конфигурации короткоживущих отрицательных ионов в состояниях трехэлектронной конфигурации $5 p^{3}$.

Улучшение монокинетичности пучка налетающих электронов и уменьшение шага измерений по энергии позволит более детально исследовать структуру ФВ и разделить вклады от разных АИС.

\section{Финиансирование работы}

Работа частично была выполнена в рамках программы INTAS (grant Ref. No 03-51-4706).

\section{Благодарности}

Авторы выражают искреннюю благодарность коллегам Г.Н. Огурцову и С.А. Шейнерману (Россия) за полезное обсуждение ряда вопросов.

\section{Конфликт интересов}

Авторы заявляют, что у них нет конфликта интересов.

\section{Список литературы}

[1] Козлов М.Г. Спектры поглощения паров металлов в вакуумном ультрафиолете. М.: Наука, 1981. 264 с.

[2] Mansfield M.W.D., Murnane M.M. // J. Phys. B. 1985. V. 18. N 21. P. 4223. doi $10.1088 / 0022-3700 / 18 / 21 / 011$

[3] Moore Ch.E. // Atomic Energy Levels. Washington, Circular of the Nat. Bureau of Standards, 1958. V. 3.

[4] Predojević B., Šević D., Pejcev V., Marinković B.P., Filipović D.M. // J. Phys. B. 2003. V. 36. N 11. P. 2371. doi 10.1088/0953-4075/36/11/319

[5] Шиеник О.Б., Запесочный И.П., Совтер В.В., Контрош Е.Э., Завилопуло А.Н. // ЖЭТФ. 1974. Т. 65. № 5. C. 1797; Shpenik O.B., Zapesochnyi I.P., Sovter V.V., Kontrosh E.E., Zavilopulo A.N. // Sov. Phys. JETP. 1974. V. 38. N 5. P. 898.

[6] Эрдевди Н.М., Шпеник О.Б., Вукстич В.С. // Опт. и спектр. 2004. T. 97. № 4. C. 559; Erdevdi N.M., Shpenik O.B., Vukstich V.S. // Opt. Spectrosc. 2004. V. 97. N 4. P. 522. doi 10.1134/1.1813692

[7] Nienhuis G., Heideman H.G.M. // J. Phys. B. 1975. V. 8. N 11. P. 2225. doi 10.1088/0022-3700/8/13/014

[8] Кучиев М.Ю., Шейнерман С.А. // УФН. 1989. Т. 158. № 7. C. 353. doi 10.3367/UFNr.0158.198907a.0353; Kuchiev M.Yu., Sheinerman S.A. // Sov. Phys. Usp. 1989. V. 32. N 7. P. 569. doi 10.1070/PU1989v032n07ABEH002731 
[9] Vukstich V.S., Remeta E.Yu., Erdevdy M.M., Shpenik O.B. // Abstr. XXIV Int. Conf. on Photonic, Electronic and Atomic Collisions (ICPEAC). Rosario, Argentina. 2005. July 20-26. P. Th039.

[10] Вукстич В.С., Ремета Е.Ю., Эрдевди Н.М., Шиеник О.Б. // Опт. и спектр. 2008. Т. 104. № 4. C. 583; Vukstich V.S., Remeta E.Y., Erdevdi N.M., Shpenik O.B. // Opt. Spectrosc. 2008. V. 104. N 4. P. 524. doi 10.1134/S0030400X08040073

[11] Shpenik O.B., Erdevdy M.M., Vukstich V.S. // Ukr. J. Phys. 2005. V. 50. N 4. P. 340.

[12] Богачев Г.Г., Ремета Е.Ю. // Опт. и спектр. 2007. Т. 103. № 5. C. 733; Bogachev G.G., Remeta E.Y. // Opt. Spectrosc. 2007. V.103. N 5. P. 709. doi 10.1134/S0030400X07110045

[13] Bogachev H., Remeta E., Borovik V., Zatsarinny O. // 37th Meeting of the Division on Atomic, Molecular and Optical Physics (DAMOP 2006). Knoxville, Tennessee, USA. 16-29 May 2006. V. 51. N 3. Abstract O1.00028. P. 88.

[14] Bogachev H., Remeta E., Zatsarinny O. // Abstr. XXV Int. Conf. on Photonic, Electronic and Atomic Collisions (ICPEAC), 2007, July 25-31, Freiburg, Germany, P. We091.

[15] Алексахин И.С., Богачев Г.Г., Запесочный И.П., Угрин С.Ю. // ЖЭТФ. 1981. Т. 80. № 6. С. 2187; Aleksakhin I.S., Bogachev G.G., Zapesochnyi I.P., Ugrin S.Yu. // Sov. Phys. JETP. 1981. V. 53. N 6. P. 1140.

[16] Богачев Г.Г., Ремета Е.Ю. // Опт. и спектр. 1999. Т. 86. № 5. C. 727.

[17] Hanel G., Gstir B., Fiegele T., Hagelberg F., Becker K., Scheier P., Snegursky A., Märk T.D. // J. Chem. Phys. 2002. V. 116. N 6. P. 2456. doi 10.1063/1.1428341

[18] Barker R.B., Berry H.W. // Phys.Rev. 1966. V. 151. N 1. P. 14. doi 10.1103/PhysRev.151.14

[19] Pejcev V., Ross K.J., Rassi D., Ottley T.W. // J. Phys. B. 1977. V. 10. N 3. P. 459. doi 10.1088/0022-3700/10/3/014 